\title{
Effects of Repetitive High Frequency Transcutaneous Electrical Nerve Stimulation (HF-TENS) on Spasticity and Motor Function following Spinal Cord Injury in Rats
}

\author{
HWI-YOUNG CHO, PT, MSc ${ }^{1, *}$, EUN-HYE KIM, PT, MSc ${ }^{2, *}$, BOKKYU KIM, PT, MS ${ }^{2)}$, \\ Go-eUn LeE, PT, MSc ${ }^{2)}$, SEOK-CHAN HAHM, PT, MSc ${ }^{2)}$, GYU-CHANG LEE, PT, $\mathrm{PhD}^{2,3}$, \\ Young WoOK Yoon, MD, PhD $^{1)}$, JunESUN KIM, PT, PhD $^{2)}$ \\ 1) Department of Physiology, Korea University College of Medicine \\ 2) Department of Physical Therapy, Korea University College of Health Science: 1 Jungneung 3 dong, \\ Sungbuk-gu, Seoul, 136-703 Republic of Korea.TEL: +82 2-940-2834,E-mail: junokim@korea.ac.kr \\ 3) Department of Physical Therapy, Kyungnam University College of Natural Science \\ *) Two authors contributed equally to this work.
}

\begin{abstract}
Purpose] The aim of this study was to determine whether high frequency transcutaneous electrical nerve stimulation (HF-TENS) influences spasticity and motor function recovery after spinal cord injury (SCI). [Subjects] Thirty six male Sprague-Dawley rats (200-250 g) were received contusive SCI at T10 level. One week after SCI, 20 rats with SCI showed increased spasticity. Rats with spasticity after SCI were randomly assigned into two groups: the HF-TENS group $(\mathrm{n}=10)$ and the control group $(\mathrm{n}=10)$. [Methods] HF-TENS stimulation $(100 \mathrm{~Hz}, 200 \mu \mathrm{s})$ was applied to anterior tubercle of the tibia near the knee joint and ankle joint in front of the achilles tendon. To test the effects of HF-TENS on spasticity and motor function recovery, we assessed BBB, combined behavioral score and Modified Ashworth scale for 9 days, at 1 hour after HF-TENS application on hindlimb. [Results] Rats with HF-TENS showed a significant decrease in spasticity 7 days after the HF-TENS application and a prominent improvement in functional outcome 5 days after the HF-TENS application compared to the control group. [Conclusion] Our results suggest that HF-TENS may help relieve spasticity and improve recovery of motor function after SCI.

Key words: Spinal cord injury (SCI), Spasticity, Transcutaneous electrical nerve stimulation (TENS), Motor function
\end{abstract}

(This article was submitted Aug. 24, 2011, and was accepted Oct. 4, 2011)

\section{INTRODUCTION}

Spasticity, defined as abnormal excessive muscle tone, is a major symptom after spinal cord injury (SCI) that may contribute to the persistent problems in rehabilitation in patients with $\mathrm{SCI}^{1)}$. Spasticity can cause many serious problems, including pain, impairment of free movement ${ }^{2}$, and limitations in daily living activities ${ }^{3)}$. Several drugs such as baclofen and tizanidine usually have been used in the treating spasticity. However, many clinical trials of these spasmolytic drugs had limited success, because their side effects manifested after long usage ${ }^{4,5)}$. In spite of the best efforts to manage spasticity, it remains strong interest in the discovery of novel therapeutics for spasticity.

There are many physical therapeutic approaches to spasticity management exit, including the use of superficial cold, diathermy and electrical stimulation. Several studies have been reported the effects of transcutaneous electrical nerve stimulation (TENS) on alleviation of spasticity accompanied with neurological conditions such as stroke and multiple sclerosis in human patients ${ }^{6-8}$. It has been suggested that TENS reduced spasticity with possible mechanisms by modulating abnormal spinal inhibitory circuits in patients with $\mathrm{SCI}^{9,10)}$. Levin et al. found that repeated applications of TENS to the common peroneal nerve of the affected leg in spastic hemiparetic patients decreased spasticity with a reduction in magnitude of stretch reflexes ${ }^{11)}$. However, other studies have shown that TENS impaired motor function recovery after sciatic nerve crush injury in experimental animal models ${ }^{12)}$.

Previous studies demonstrated that high-frequency electrical stimulation was more effective than low frequency for treating in spasticity ${ }^{13-15)}$. Studies have shown high-frequency TENS (HF-TENS) application to peripheral nerve treated spasticity effectively, but most of studies for reducing spasticity conducted by the short-term effects of HF-TENS ${ }^{7,10,13,16)}$ and research on its long-term effects on spasticity of stroke is still insufficient.

Recent study showed that a single session of HF-TENS can immediately reduce spasticity following SCI in human patients ${ }^{17}$. Several other studies have shown the effects of HF-TENS on spasticity in neurological conditions, but 
cumulative effects of HF-TENS on reducing spasticity and improvement of motor function recovery after SCI are not clear. Testing the effects of repetitive HF-TENS in reducing the spasticity and improving the motor function recovery in an experimental animal model may help to resolve this controversy. We have already reported that characteristics of motor recovery after SCI using spinal contusion animal model in rats ${ }^{18)}$.

Thus, the present study investigated whether a repetitive HF-TENS application reduces spasticity of hindlimbs and improves motor function recovery after SCI in rats.

\section{SUBJECTS AND METHODS}

All animal procedures were approved by the Institutional Animal Care and Use Committee (IACUC) at the Korea University. The experiments were conducted on adult male Sprague-Dawley rats ( $\mathrm{n}=36$; Orient Bio Inc., Seoul, Korea) weighing between 200-250 g. The animals were kept in a thermo-hygrostat (temperature: $22-25{ }^{\circ} \mathrm{C}$, humidity: $55 \%$ ) under 12:12 light/dark cycle (08:00-20:00). All animals were kept for least 5 days before surgery with ad libitum access to water and food.

The contusion was performed at the T10 level using the New York University (NYU) impactor under enflurane anesthesia (a mixture of $4 \%$ enflurane and 95\% O2). A laminectomy was performed at the T10 spinal segment and the spinal column was stabilized by clamping the spinous processes of T9 and T11 with tissue forceps. A $10 \mathrm{~g}$ weight was then released onto the spinal cord from a $12.5 \mathrm{~mm}$ height. After the spinal cord contusion, the musculature was sutured, the skin was auto-clipped and the animals were allowed to recover from the anesthesia. The rats were housed four to a cage. The bladders were manually expressed twice a day until spontaneous urination had returned. To prevent urinary tract infection, Unasyn (Ampicillin/sulbactam, $100 \mathrm{mg} / \mathrm{kg}$, Pfizer, Seoul, Korea) was injected intraperitoneally once a day for the first 3-5 days. Absorbent bedding was used to prevent sores and infections in the paralyzed animals.

Following spinal contusion, rat showed increased resistant to passive movement in hindlimbs 1 week after injury: this was interpreted as spasticity. Two skin electrodes were attached to anterior tubercle of the tibia near the knee joint and the ankle joint in front of the Achilles tendon at the level of the superior border of medial malleolus. HF-TENS stimulation (Intelect NMES, Chattanooga group Inc., USA; frequency $100 \mathrm{~Hz}$, pulse width $200 \mu \mathrm{s}$ ) was started 7 days postoperatively (PO) and applied for 15 minutes per a day as a single session, for a total of 10 sessions. Whereas the control group $(n=10)$ was received attachement of skin electrodes without electric delivery. Applied intensity was determined by increasing the intensity until a visible muscle contraction was observed and then diminishing the intensity to just below this level. This intensity was between $1.5 \mathrm{~mA}$ and $4 \mathrm{~mA}$ for most animals. Therefore, the intensity started at $1 \mathrm{~mA}$, gradually increased to a maximum $4 \mathrm{~mA}$.

To exclude the short-term effects of HF-TENS, Behavioral tests for resistance to passive movement and motor function were conducted 1 hour after HF-TENS application. The tests were performed on each rat: 1 day prior to surgery; 1, 3, 5, 7 and 9 days PO before the start of the experiments. The rats that showed slight spasticity were excluded from the study. Other interventions except HF-TENS and medications were not provided during the conducting experiment.

For measuring motor function recovery after HF-TENS application, two methods were used. First, locomotor function was assessed by using the Basso, Beattie, and Bresnahan (BBB) locomotor Rating Scale ${ }^{19}$. Briefly, the $\mathrm{BBB}$ is divided into 22 levels from 0 to 21 .

0-7: the early phase of recovery with the return of isolated joint movements of the three joints including hip, knee, and ankle

8-13: the intermediated phase of recovery with the return of paw placement, stepping and forelimb-hindlimb coordination

14-21: the late phase of recovery with the return of toe clearance during the stepping, predominant paw position, trunk stability and tail position. Left and right hindlimbs were assessed separately due to asymmetrical recovery.

Second, a modification of the combined behavioral score (CBS) of Gale et al. ${ }^{20)}$ was performed at the time of the behavioral test for motor function. The CBS assigns a weight to each of its tests and combines them into one total score that represents the degree of motor impairment. Tests were as follows; motor scores, toe spread, righting reflex, extension withdrawal reflex, placing reflex and inclined plane. Neurological function was evaluated by a scoring system that is ranging from 0 for a normal rat to 90 for a completely paralyzed rat.

The resistance to the passive movement assessed by using modified Ashworth score (MAS). MAS rates passive movement resistance as follows; 0 , no increase in muscle tone; 1 , slight increase in tone giving a 'catch' when the limb is moved in flexion or extension; $1+$, slight increase in tone giving a 'catch' followed by minimal resistance throughout the remainder of the range of motion (ROM); 2 , more marked increase in tone but the limb is easily flexed; 3 , considerable increase in tone-passive movement difficult; 4 , limb rigid in flexion or extension. This study measured spasticity in grades of $0,1,2,3,4$ and 5 , with the mean found after repeating the measurement for three times. All the examinations were performed in a blind manner. To evaluate MAS scale, all rats were trained 1 week before beginning the experiments to adapt animals to environment and the procedures. Rats took the posture of prone position during the executing experiments. Measurer held the head of rat and covered rat's eyes. After rats were habituated the environment and then relaxed completely, the passive movements were given to the hindlimbs of rats ${ }^{21)}$. Generally, rats did not show any particular motions in other parts of body other than bending motion in the involved extremities when executed the passive movements in hindlimbs. When rats showed any signs of struggling, enough time to be relaxed was provided.

Statistical analyses were performed using SPSS (v 15). All values were expressed as mean \pm standard error of the mean (SEM). The Mann-Whitney U test was used to 
Table 1. Comparison of the changes in the BBB, CBS and MAS scores of the HF- TENS group and control group

\begin{tabular}{llllllll}
\hline Measurements & \multicolumn{1}{c}{ Groups } & \multicolumn{1}{c}{ Pre } & \multicolumn{1}{c}{ 1D } & \multicolumn{1}{c}{ 3D } & \multicolumn{1}{c}{ 5D } & 7D & 9D \\
\hline BBB score & HF-TENS $(\mathrm{n}=10)$ & $7.84 \pm 1.03$ & $8.12 \pm 0.74$ & $11.16 \pm 1.03$ & $12.56 \pm 0.68^{*}$ & $13.21 \pm 0.79^{*}$ & $13.65 \pm 0.92^{*}$ \\
& Control $(\mathrm{n}=10)$ & $7.57 \pm 0.87$ & $7.87 \pm 1.06$ & $10.25 \pm 0.89$ & $10.74 \pm 0.83$ & $10.36 \pm 0.88$ & $11.73 \pm 0.67$ \\
\hline CBS score & HF-TENS $(\mathrm{n}=10)$ & $39.68 \pm 4.26$ & $35.13 \pm 4.13$ & $27.58 \pm 3.62^{*}$ & $25.66 \pm 4.12^{*}$ & $22.73 \pm 3.68^{*}$ & $22.52 \pm 3.08^{*}$ \\
& Control $(\mathrm{n}=10)$ & $41.25 \pm 3.57$ & $36.38 \pm 3.62$ & $34.37 \pm 3.73$ & $31.63 \pm 2.68$ & $28.27 \pm 2.63$ & $29.77 \pm 3.13$ \\
\hline \multirow{2}{*}{ MAS score } & HF-TENS $(\mathrm{n}=10)$ & $3.10 \pm 0.19$ & $2.75 \pm 0.25$ & $2.50 \pm 0.19$ & $2.45 \pm 0.16$ & $2.16 \pm 0.18^{*}$ & $1.75 \pm 0.25^{*}$ \\
& Control $(\mathrm{n}=10)$ & $3.15 \pm 0.32$ & $3.00 \pm 0.19$ & $2.85 \pm 0.27$ & $2.75 \pm 0.20$ & $2.63 \pm 0.22$ & $2.45 \pm 0.17$ \\
\hline
\end{tabular}

Note. Values are expressed as mean \pm SEM. * means a significant difference compared with the control group at the same time point (p<0.05). HFTENS: high frequency Transcutaneous Electrical Nerve Stimulation; BBB: the Basso, Beattie, and Bresnahan locomotor Rating Scale; CBS: the combined behavioral score; MAS: Modified Ashworth scale.

compare scores for the BBB, CBS and MAS scores obtained on a given experimental day between two groups. $P$ values less than 0.05 were considered to be significant.

\section{RESULTS}

All rats were evaluated locomotor function in both hindlimbs using the BBB score by open field test. Prior to the contusion injury, all rats showed normal gait with a $\mathrm{BBB}$ score of 21 . Immediately after the spinal contusion, the rats showed paralysis in their hindlimbs, corresponding to a BBB score of 0 . At 1 day after the contusion injury, their BBB scores decreased markedly to $1.8 \pm 0.5$. However, progressive motor recovery was observed with hindlimb joint movement at 4 days PO and then relatively rapid recovery preceded until 14 days PO. The CBS scores increased to $68.6 \pm 1.8$ at 1 day PO, whereas the CBS score of all rats before the injury was 0 and dropped to $42.8 \pm 2.3$ at 7 days PO. By 3 weeks after the SCI, motor recovery gradually proceeded (data not shown). These results are consistent with our previous report ${ }^{18)}$.

To test the effect of repetitive HF-TENS on motor function recovery after SCI, HF-TENS was applied from 7 days PO after SCI. There were no significant differences in BBB and CBS score between the HF-TENS and control groups. After the HF-TENS application, we found no significant difference in the BBB scores in either the HF-TENS or control groups, for the first 3 days. However, as of 5 days after the HF-TENS group began application, rats showed a prominent recovery of locomotor function compared to the controls. There were statistically significant differences between the groups (Table 1, p<0.05). Functionally prominent difference in locomotor function between the HF-TENS and controls was noticed up to 9 days after HF-TENS application when its application was ended.

Although this score difference did not seem great, the difference in terms of motor functional gain was significant. Rats in the control group exhibited frequent weightsupported plantar stepping, with no forelimb-hindlimb coordination. In contrast, the rat with HF-TENS application showed consistent weight-supported plantar steps and consistent forelimb-hindlimb coordination.

Simultaneously, CBS score were measured on 1, 3, 5, 7 and 9 days after the HF-TENS application began at 7 days PO after SCI. After the repeated HF-TENS, rats showed significant decrease in CBS scores. Similar to the locomotor function results, significant difference in CBS score appeared from 3 days and maintained up to 9 days after HF-TENS application (Table 1, $\mathrm{p}<0.05$ ).

Modified Ashworth score (MAS) was measured in both hindlimbs before and after the spinal contusion. MAS score started to go up from 4 days PO and gradually increased by 1 week PO and were maintained in the high level up to 3 weeks after the SCI, where allowed achievement of maximal motor recovery. To test the effect of HF-TENS on spasticity, MAS score was measured for 9 days in the HF-TENS and control group at 7 days PO after SCI (Table $1, \mathrm{p}<0.05)$. Rats subjected to contusive spinal cord injury showing MAS score above 2 were used in present study. In the HF-TENS group, MAS score decreased slightly compared to control beginning 1 day after HF-TENS application. The prominent decrease of spasticity was observed from 7 days after HF- TENS application began.

\section{DISCUSSION}

This study demonstrated that repeated HF-TENS application reduced spasticity following SCI. We also found that repeated HF-TENS application improved reflex and locomotor function. As several previous studies, which showed the beneficial effects of TENS on spasticity, suggest ${ }^{6-8)}$, the present study indicates HF-TENS possesses therapeutic potential for controlling spasticity after SCI. To our knowledge, this is the first study evaluating the cumulative effects of long-term HF-TENS application on both spasticity and motor function in SCI using an animal model.

Spasticity, a feature of upper motor neuron syndrome, is defined as abnormal excessive muscle tone. Spasticity can raise problem in patients' activities of daily living, because it interferes with voluntary motor control and causes muscle tightness, which in be discouraged the progress of motor function recovery ${ }^{1,2)}$.

In this study, we used as contusive experimental animal model by using the NYU impactor which is one of accepted contusion models for studying SCI, because it generates injuries closely paralleling human clinical SCI cases ${ }^{22}$. In addition, we examined spasticity and motor function after the contusive SCI to test the HF-TENS effects on spasticity and motor recovery. Immediately after the contusive SCI, the rats showed paralysis in both hindlimbs, but motor 
function progressively returned from 4 days PO. With this progressive motor recovery, spasticity was observed in the hindlimbs and gradually increased above 2 of MAS score and maintained up to 3 weeks PO where reached plateau of motor recovery after SCI. Similar to the present results, Dong et al. ${ }^{21)}$ reported that spasticity developed from 4 days and reached its peak on 7 days and maintained until 56 day after SCI in the spinal compression model.

To determine the therapeutic effects of HF-TENS on spasticity and motor recovery, HF-TENS administration was started 7 days after SCI in this study. The present results showed that HF-TENS application improved the recovery of motor function with progressive return of the reflexes compared to the control group. As a result, the significant difference in locomotor function between the HF-TENS and the control groups occurred beginning 5 days after HF-TENS application. However, the prominent motor functional difference between the HF-TENS and control groups appeared from 7 days after HF-TENS application. The two groups showed a sizeable difference in hindlimb function quality at that time point, although there was no great difference in the BBB scores, expressed in numbers. Rat receiving placebo-TENS application (control group) merely placed their limbs in a weighted fashion and showed occasional weighted plantar steps, whereas the HF-TENS group displayed nearly consistent plantar stepping with frequent forelimb-hindlimb coordination.

HF-TENS seems to be effective for reducing spasticity in the human and experimental SCI models including in our current results. HF-TENS slightly reduced spasticity from 1 day after HF-TENS began; however, the significant effects of reducing spasticity appeared starting 7 days after HF-TENS began. The time course of these therapeutic effects on spasticity is similar to that of motor recovery. It suggests that alleviation of spasticity due to HF-TENS is correlated with improvement in reflex and voluntary motor functions. Our results are consistent with the previous report $^{21)}$ where effect of HF-TENS on spasticity was observed after compressive SCI. They found that $100 \mathrm{~Hz}$ TENS applied on near the knee joint effectively reduced spasticity immediately after the TENS stimulation. Similarly an earlier study on spastic hemiparetic patiens ${ }^{11)}$ which showed that repeated TENS applications to peripheral nerve of the affected leg decreased spasticity with a reduction in stretch reflex magnitude, strongly supports our results.

After SCI, Ia-reciprocal inhibition is decreased. The treatment-induced activation of the Ia-reciprocal inhibition pathway $^{23)}$ and stimulation of the cutaneous afferents, which could repress motoneuronal excitability by depressing the propriospinal inter-neurons or inducing long term synaptic changes in primary afferents in the dorsal horn $^{24)}$ might be responsible for the spasticity reduction. Previous studies showed that high-frequency electrical stimulation is more effective for treating spasticity than is low frequency ${ }^{13-15)}$. It has been suggested that the HF-TENS acts to reduce spasticity by modulating abnormal spinal inhibitory circuits ${ }^{9,10)}$ by releasing dynorphin A and GABA. Recent studies demonstrated that HF-TENS causes the release of spinal dynorphin ${ }^{21)}$, and spinal GABA ${ }^{25)}$.
Taken together, these findings also strongly suggest HF-TENS has therapeutic potential for treating spasticity and aiding motor recovery after certain types of SCI.

The present study showed that HF- TENS reduced spasticity and improved locomotor recovery after SCI in rats. These results suggest HF-TENS is useful in controlling spasticity and improving motor recovery following SCI. Further studies are warranted to investigate the mechanisms of this effect to provide evidence for the clinical application of HF-TENS.

\section{ACKNOWLEDGEMENT}

This work was supported by the Basic Science Research Program through the National Research Foundation of Korea (NRF) funded by the Ministry of Education, Science and Technology (MEST) 2010-0006489.

\section{REFERENCES}

1) Decq P: [Pathophysiology of spasticity]. Neurochirurgie, 2003, 49: 163-184.

2) Meythaler JM, Guin-Renfroe S, Hadley MN: Continuously infused intrathecal baclofen for spastic dystonic hemiplegia - A preliminary report. Am J Phys Med Rehab, 1999, 78: 247-254.

3) Lundqvist $C$, Siosteen $A$, Blomstrand $C$, et al.: Spinal-Cord Injuries Clinical, Functional, and Emotional Status. Spine, 1991, 16: 78-83.

4) Delwaide PJ, Pennisi G: Tizanidine and electrophysiologic analysis of spinal control mechanisms in humans with spasticity. Neurology, 1994, 44: S21-S28.

5) Loubser PG, Narayan RK, Sandin KJ, et al.: Continuous infusion of intrathecal baclofen: long-term effects on spasticity in spinal cord injury. Paraplegia, 1991, 29: 48-64.

6) Joodaki MR, Olyaei GR, Bagheri H: The effects of electrical nerve stimulation of the lower extremity on H-reflex and F-wave parameters. Electromyogr Clin Neurophysiol, 2001, 41: 23-28.

7) Potisk KP, Gregoric M, Vodovnik L: Effects of transcutaneous electrical nerve stimulation (TENS) on spasticity in patients with hemiplegia. Scand J Rehabil Med, 1995, 27: 169-174.

8) Armutlu K, Meric A, Kirdi N, et al.: The effect of transcutaneous electrical nerve stimulation on spasticity in multiple sclerosis patients: a pilot study. Neurorehab Neural Re, 2003, 17: 79-82.

9) Crone C, Nielsen J, Petersen N, et al.: Disynaptic Reciprocal Inhibition of Ankle Extensors in Spastic Patients. Brain, 1994, 117: 1161-1168.

10) Perez MA, Field-Fote EC, Floeter MK: Patterned sensory stimulation induces plasticity in reciprocal ia inhibition in humans. J Neurosci, 2003, 23: 2014-2018.

11) Levin MF, Huichan CWY: Relief of hemiparetic spasticity by tens is associated with improvement in reflex and voluntary motor functions. Electroen Clin Neuro, 1992, 85: 131-142.

12) Gigo-Benato D, Russo TL, Geuna $S$, et al.: Electrical stimulation impairs early functional recovery and accentuates skeletal muscle atrophy after sciatic nerve crush injury in rats. Muscle Nerve, 2010, 41: 685-693.

13) Goulet $C$, Arsenault AB, Bourbonnais D, et al.: Effects of transcutaneous electrical nerve stimulation on H-reflex and spinal spasticity. Scand J Rehabil Med, 1996, 28: 169-176.

14) Liss S, Liss B: Physiological and therapeutic effects of high frequency electrical pulses. Integr Physiol Behav Sci, 1996, 31: 88-95.

15) Sonde L, Gip C, Fernaeus SE, et al.: Stimulation with low frequency (1.7 $\mathrm{Hz}$ ) transcutaneous electric nerve stimulation (low-tens) increases motor 
function of the post-stroke paretic arm. Scand J Rehabil Med, 1998, 30 95-99.

16) Wang RY, Chan RC, Tsai MW: Effects of thoraco-lumbar electric sensory stimulation on knee extensor spasticity of persons who survived cerebrovascular accident (CVA). J Rehabil Res Dev, 2000, 37: 73-79.

17) Ping Ho, Chung B, Kam Kwan Cheng B: Immediate effect of transcutaneous electrical nerve stimulation on spasticity in patients with spinal cord injury. Clin Rehabil, 2010, 24: 202-210.

18) Jung JI, Kim J, Hong SK, et al.: Long-term Follow-up of Cutaneous Hypersensitivity in Rats with a Spinal Cord Contusion. Korean J Physiol Pha, 2008, 12: 299-306.

19) Basso DM, Beattie MS, Bresnahan JC: A sensitive and reliable locomotor rating scale for open field testing in rats. J Neurotrauma, 1995, 12: 1-21.

20) Gale K, Kerasidis H, Wrathall JR: Spinal cord contusion in the rat: behav- ioral analysis of functional neurologic impairment. Exp Neurol, 1985, 88: 123-134.

21) Dong HW, Wang LH, Zhang M, et al.: Decreased dynorphin A (1-17) in the spinal cord of spastic rats after the compressive injury. Brain Res Bull, 2005, 67: 189-195.

22) Hulsebosch CE, Xu GY, Perez-Polo JR, et al.: Rodent model of chronic central pain after spinal cord contusion injury and effects of gabapentin. $\mathrm{J}$ Neurotrauma, 2000, 17: 1205-1217.

23) Kuo JJ, Lee RH, Johnson MD, et al.: Active dendritic integration of inhibitory synaptic inputs in vivo. J Neurophysiol, 2003, 90: 3617-3624.

24) Dewald JP, Given JD, Rymer WZ: Long-lasting reductions of spasticity induced by skin electrical stimulation. IEEE Trans Rehabil Eng, 1996, 4: 231-242.

25) Maeda Y, Lisi TL, Vance CG, et al.: Release of GABA and activation of GABA(A) in the spinal cord mediates the effects of TENS in rats. Brain Res, 2007, 1136: 43-50. 